\title{
Irrelevant speech eliminates the word length effect
}

\author{
IAN NEATH and AIMÉE M. SURPRENANT \\ Purdue University, West Lafayette, Indiana \\ and \\ DENNY C. LECOMPTE \\ Louisiana State University, Baton Rouge, Louisiana
}

\begin{abstract}
The word length effect refers to the observation that memory is better for short than for long words. The irrelevant speech effect refers to the finding that memory is better when items are presented against a quiet background than against one with irrelevant speech. According to Baddeley's (1986, 1994) working memory, these variables should not interact: The word length effect arises from rehearsal by the articulatory control process, whereas irrelevant speech reduces recall through interference in the phonological store. Four experiments demonstrate that, like articulatory suppression, irrelevant speech eliminates the word length effect for both visual and auditory items. These results (1) provide further evidence against the ability of working memory to explain the word length and irrelevant speech effects and (2) confirm a specific prediction of Nairne's (1990) feature model.
\end{abstract}

First identified by Mackworth (1963), the word length effect refers to the finding that people can remember short words (items that take less time to pronounce) better than long words. This effect is observable with both free (M. J. Watkins, 1972) and serial (M. J. Watkins \& O. C. Watkins, 1973) recall, for both auditory and visual items (M. J. Watkins \& O. C. Watkins, 1973), can be found when the items are equated for number of syllables and word frequency (Baddeley, Thomson, \& Buchanan, 1975 ), and is even observable when the phonemic components of the long and short items are closely matched (Cowan et al., 1992). There are two well-developed explanations of the word length effect, Baddeley's (1986, 1992, 1994) working memory ${ }^{1}$ and Nairne's (1988, 1990) feature model. In this paper, we compare the predictions of each view concerning interactions between word length and irrelevant speech.

\section{Working Memory}

Baddeley's $(1986,1992,1994)$ model of working memory (or WMM) is a specific theory of how memory operates which consists of three basic components. The central executive is a controlling attentional mechanism that coordinates the activity of a number of subsidiary slave systems, including the visuospatial sketchpad and the phonological loop. The visuospatial sketchpad is concerned with visual imagery and processing spatial infor-

We thank Rebecca Mazzorana for assistance testing subjects. Correspondence may be addressed to I. Neath (neath@psych.purdue.edu) or A. M. Surprenant (aimee@psych.purdue.edu) at 1364 Psychological Sciences Building, Purdue University, West Lafayette, IN 47907-1364. mation, whereas the phonological loop is concerned with processing verbal information. Two components make up the phonological loop: the phonological store and the articulatory control process. The phonological store retains speech-based information for a short period of time: traces within the store are assumed to decay within about 1-2 sec, after which they are no longer usable. Auditory information has direct access to the phonological store because it is already in the appropriate code, but visual information has to be translated before it can enter. The second component of the phonological loop is the articulatory control process, which has two main functions. First, it translates visual information into a speech-based code so that the information can enter the phonological store. Second, it refreshes decaying traces within the phonological store to offset the time-based decay process. If rehearsal of a particular item does not occur within a certain amount of time, the memory trace for that item will have decayed too far to be usable. The amount of verbal information that can be retained is therefore a tradeoff between the decay rate and the covert rehearsal rate.

This current version of the WMM was designed, according to Baddeley $(1986,1992,1994)$, to account for four basic phenomena of immediate memory: the phonological similarity effect, the word length effect, the irrelevant speech effect, and the effects of articulatory suppression. The phonological similarity effect refers to the finding (Conrad, 1964) that memory is worse for items that sound alike (PGTCD) than for items that differ (RHXKW). Of more concern in this paper are how the last two phenomena interact with word length.

The word length effect is explained within the WMM by assuming that the time needed to rehearse an item using the articulatory control process is related to the 
time needed to pronounce the item. Given this assumption, the shorter the items in terms of pronunciation time, the greater the number of items that can be covertly rehearsed before the traces decay. One way to test this explanation is to perform a manipulation that prevents the subject from engaging the articulatory control process.

When subjects engage in articulatory suppression, they repeatedly say a word such as the over and over out loud (Murray, 1968). According to the WMM, articulatory suppression occupies the articulatory control process and causes two specific results: (1) Visually presented items can no longer be translated into a speech-based code and thus cannot enter the phonological store, and (2) covert rehearsal of items in the phonological store cannot take place. Articulatory suppression, then, removes the key process responsible for observing the word length effect--subvocal rehearsal-and the prediction is that the word length effect will be eliminated.

When subjects engage in articulatory suppression during list presentation, the word length effect is eliminated for visual but not for auditory items (Baddeley et al., 1975). For auditory items, articulatory suppression must continue throughout recall to remove the effect of word length (Baddeley \& Lewis, 1984; Baddeley, Lewis, \& Vallar, 1984). The reason, according to Baddeley (1986), is that subjects may be rehearsing during recall, refreshing the decaying trace in the phonological store while simultaneously recalling items. This is more likely for auditory items, because they are automatically registered in the phonological store and do not require conversion using the articulatory control process during presentation. To the extent that subvocal rehearsal is prevented during both presentation and recall, there will be no word length effect, just as the WMM predicts (Baddeley, 1992).

The irrelevant speech effect refers to the finding that memory is impaired if presentation of the to-be-remembered items is accompanied by auditory presentation of irrelevant speech (Colle \& Welsh, 1976). According to the WMM (Salamé \& Baddeley, 1982), the phonemes from the irrelevant speech enter the phonological store automatically and interfere with the information about the to-be-remembered items. This produces an overall decrement in performance, reminiscent of the phonological similarity effect. Irrelevant speech does not affect the articulatory control process and should therefore have no effect on manipulations involving word length.

The key theoretical point is that whereas articulatory suppression affects the articulatory control process, irrelevant speech "impairs performance by corrupting the phonological store" (Baddeley, 1986, p. 90). A clear prediction from the WMM (Baddeley, 1986, 1992, 1994), then, is that irrelevant speech will not eliminate the word length effect. Although irrelevant speech may lower overall performance because of some corruption of traces within the phonological store, it will have no effect on the articulatory control process. The latter process is still available to rehearse the traces in the phonological store, so that short items should still have an advantage over long items.

\section{The Feature Model}

Whereas the central forgetting process in Baddeley's $(1986,1992,1994)$ WMM is time-based decay, the central forgetting process in Nairne's $(1988,1990)$ feature model is interference. The feature model gets its name from the assumption that memory traces are made up of two types of features. Modality-dependent features represent the conditions of presentation, including presentation modality, whereas modality-independent features represent the nature of the item itself and are generated through internal processes such as categorization and identification. At the end of list presentation, primary memory will consist of partially degraded representations of the list items. Degradation is due entirely to retroactive interference. The task at recall is to match the degraded primary memory traces to the correct undegraded secondary memory traces. In the feature model, primary memory has no capacity limits-whether time based or item based-and does not employ the concept of decay.

For each partially degraded trace in primary memory, the subject tries to select an appropriate recall candidate by comparing the degraded trace with intact traces in the secondary memory search set. The probability of matching a particular secondary memory trace with a particular degraded primary memory trace is described by a similarity-based choice rule (Luce, 1963). In general, the secondary memory item with the fewest mismatching features will be selected as the candidate for recall. Thus, the secondary memory trace chosen as the response for a particular degraded primary memory trace will typically be the trace with the largest proportion of matching features, relative to the other choices currently available in the secondary memory search set. The absolute number of features is irrelevant; far more important is the relative proportion of matching features (Neath \& Nairne, 1995).

The feature model explains the word length effect in a way suggested by Melton (1963). The basic idea is that the more parts there are, the more opportunities there are to make a mistake. To take an extreme example, a jigsaw puzzle with only 2 pieces is easier to assemble than a puzzle with 10 pieces, which in turn is easier to assemble than a puzzle with 100 pieces. Just as a list can be divided into items, an item can be divided into segments. The feature model assumes that long words have more segments than short words and that there is a certain probability of assembling a segment incorrectly (Neath \& Nairne, 1995; see Brown \& Hulme, 1995, for a similar idea). Because word length effects are seen with both auditory and visual presentation modalities, segmental assembly errors affect modality-independent features (features that are common to both presentation modalities).

When subjects engage in articulatory suppression, they repeatedly say a constant item out loud. In the fea- 
ture model (Nairne, 1990), this constant piece of information is incorporated into the memory trace of each individual item. Because articulatory suppression reduces performance for both auditory and visual items, articulatory suppression is implemented by setting some of the modality-independent features of each item to a constant value. This has the net result of increasing the similarity of the items in primary memory and decreasing the probability that a degraded trace will be matched with the appropriate undegraded trace.

According to the feature model, the word length effect is not seen under articulatory suppression because both of these manipulations alter modality-independent features. Because so many modality-independent features are modified by articulatory suppression, the effects due to a segment assembly error cannot be detected, and so the word length effect is masked. Note that according to this explanation, the word length effect is eliminated under articulatory suppression because suppression has already altered the same features that a segment assembly error will alter (see Neath \& Nairne, 1995, especially Table 4, for specific examples and simulation results).

Phonological similarity effects fall out naturally from the model. On the average, there will always be some similarity between adjacent items; phonological similarity is modeled by guaranteeing a certain number of similar features. Because sampling probability is conceived as the ratio of similarities, any increase in similarity increases the value of the denominator relative to the numerator, resulting in overall worse performance (see Nairne, 1990). The feature model also correctly predicts the interaction between modality, phonological similarity, and articulatory suppression (see Demonstration 4 of Neath \& Nairne, 1995). The phonological similarity effect is removed for visual items by articulatory suppression, whereas the effect remains for auditory items (albeit reduced; Cowan, Cartwright, Winterowd, \& Sherk, 1987; Longoni, Richardson, \& Aiello, 1993).

The feature model has not formally been applied to the irrelevant speech effect, although a preliminary analysis suggests that some of the effects of irrelevant speech can be modeled in the same way as the effects due to articulatory suppression (Surprenant, LeCompte, \& Neath, 1997; see Macken \& Jones, 1995, for a similar suggestion). According to the feature model, articulatory suppression results in a memory trace that includes some features of the irrelevant item that the subject is articulating; an irrelevant speech manipulation results in a memory trace that includes some of the features of the irrelevant item that the subject is hearing. In both cases, the addition of features from the irrelevant item-whether spoken or heard-increases the likelihood of a mismatch, and thereby reduces recall. There appear to be only two important differences between these manipulations. First, an articulatory suppression manipulation can use only speech sounds that the subject is capable of pro- ducing, whereas tones and speech tokens from other speakers and languages can be used with irrelevant speech. Second, the subject has to attend to the item with articulatory suppression (if only to the extent that attention is required to produce the item). With an irrelevant speech manipulation, the subject does not necessarily have to attend to the irrelevant items. When an irrelevant speech manipulation uses similar items as an articulatory suppression manipulation, the effects should also be similar. Because irrelevant speech and articulatory suppression are modeled in the same way, the feature model predicts that they will have similar effects, and thus it predicts that both will eliminate the word length effect.

Given that the WMM (Baddeley, 1986, 1992, 1994) predicts that irrelevant speech will not eliminate the word length effect and that the feature model (Neath \& Nairne, $1995)$ predicts that irrelevant speech will eliminate the word length effect, four experiments were conducted to see which prediction would be supported. ${ }^{2}$ In the first three experiments, we used a reconstruction of order test rather than a serial recall test, because it controls for differential output times. Cowan et al. (1992) have shown that the time required to produce (in their case, vocalize) a response can affect overall level of recall and that this has a large impact on the magnitude of the word length effect. With a reconstruction of order task, the subject simply clicks on a button, and the time needed in order to make this response should be largely independent of the length of the word displayed on the button. In Experiment 1 , we compared performance on short and long items when the background was quiet, when it contained irrelevant tones or irrelevant speech, or when the subject engaged in articulatory suppression. In Experiment 2, we tested whether the number of irrelevant speech items was important, and in Experiment 3, whether the word length effect for auditory items was eliminated by irrelevant speech. Finally, in Experiment 4, we assessed whether the effect held in a paradigm more typically used by Baddeley and his colleagues.

\section{EXPERIMENT 1}

In Experiment 1, we sought to determine whether the word length effect for visual items would be eliminated when irrelevant speech accompanied list presentation. The word length effect for visual items is eliminated when subjects engage in articulatory suppression during presentation (Baddeley et al., 1975). According to the WMM, this is because subvocal rehearsal is prevented by the articulatory suppression. Because irrelevant speech does not prevent subvocal rehearsal, the WMM predicts that the word length effect will remain. According to the feature model, the effect of irrelevant speech can be seen as similar to the effect of articulatory suppression (Surprenant et al., 1997). In both cases, features from the irrelevant item (either articulated or merely heard) are in- 
corporated into the primary memory vector representing the to-be-remembered item. The addition of these features increases the likelihood of a mismatch and thereby reduces recall. Because irrelevant speech and articulatory suppression are modeled in the same way, the feature model predicts that they will have similar effects. In particular, the feature model predicts that an irrelevant speech manipulation will abolish the word length effect just like an articulatory suppression manipulation.

\section{Method}

Subjects. Eighty Purdue University undergraduates volunteered to participate in exchange for credit in introductory psychology courses. All were native speakers of American English and each was arbitrarily assigned to one of four conditions

Stimuli. The to-be-remembered stimuli comprised 81 long and 81 short words from LaPointe and Engle (1990). Each word occurred three times throughout the experiment. During list presentation, each word was displayed in the center of an Apple computer screen for $1 \mathrm{sec}$ in black against a white background in 18-point bold Geneva font.

Design. The four between-subjects conditions were used to examine the effect of various kinds of irrelevant information. In the articulatory suppression condition, the subjects were asked to repeatedly say the word teatime out loud over and over. In the irrelevant speech condition, the subjects listened to a tape on which the word teatime was repeated over and over. In the irrelevant tone condition, the subjects listened to a tape on which two sine-wave tones-roughly corresponding to the duration and change in frequency of the spoken word teatime -were repeated over and over. In the quiet condition, there was no irrelevant information. Word length was manipulated within subjects. Sixty presentation lists were generated separately for each subject. The lists were divided into three blocks of 20 lists. Within each block, 10 lists comprising 8 items each were created by sampling randomly and without replacement from the 81 long words; the other 10 lists, also comprising 8 items each, were created by sampling randomly and without replacement from the $\mathbf{8 1}$ short words. Short and long lists were ordered randomly within each block. For the subjects in the quiet condition, all 60 lists were presented without irrelevant speech; for the subjects in the irrelevant speech condition, all 60 lists were presented with irrelevant speech; and so on for the two other conditions. The subjects were unaware of the other conditions.

Procedure. Subjects were tested individually or in pairs. Each sat in front of an Apple Macintosh IIci or PowerPC 6100 computer The subjects in the irrelevant speech and irrelevant tone conditions wore headphones. All subjects were informed that they would see lists of words and following each list would be tested on how accurately they could remember the order in which these words had been presented. For the subjects in the irrelevant speech and irrelevant tone conditions, the stream of irrelevant auditory stimuli began prior to presentation of the first list and continued uninterrupted until the subject had completed the experiment. The subjects in the articulatory suppression condition were asked to begin articulation when a warning signal was seen and to stop when the reconstruction of order test appeared.

At the end of each list, the eight list items appeared simultaneously in alphabetical order. They were arranged horizontally in the center of the screen in 12-point Chicago font. The subject's task was to use the mouse to click on the words in the same order as that in which they had appeared on the computer screen. All subjects reported having used a mouse before, and no subject reported any difficulty with this mode of responding. Twenty seconds were allowed for the test; the next list began 2 sec after the end of the test

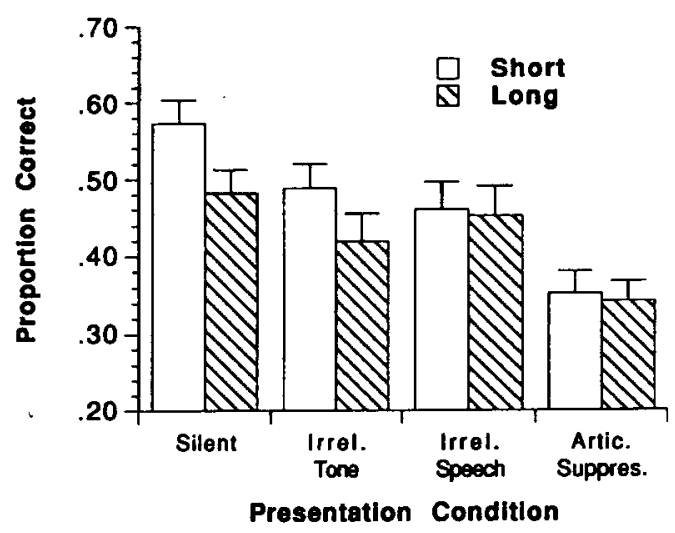

Figure 1. Proportion of short and long words correctly recalled in a serial reconstruction of order task as a function of background condition (quiet, irrelevant tones, irrelevant speech, or articulatory suppression) with visual presentation in Experiment 1. Error bars show the standard error of the mean.

period. The experiment proper was preceded by a single practice list comprising eight digits (1-8) presented in the appropriate condition (e.g., with articulatory suppression, with irrelevant tones, etc.).

\section{Results}

The main results of interest are shown in Figure 1. First, there is a reliable word length effect in the quiet condition: The proportion of short words correctly recalled in order was .572 compared to .482 for long words $[F(1,19)$ $\left.=22.71, M S_{\mathrm{e}}=0.029, p<.01\right]$. In the irrelevant tone condition, there was also a reliable word length effect, although overall performance was impaired relative to the quiet condition: The proportion of short words correctly recalled was .489 ; the proportion of long words, $.420\left[F(1,19)=12.49, M S_{\mathrm{e}}=0.031, p<.01\right]$.

This reliable word length effect was eliminated by articulatory suppression: The proportion of short words correctly recalled was .352 compared to .342 for long words $[F(1,19)<1]$. These results replicate the findings commonly seen with serial recall tests. Of more interest, however, is the finding that like articulatory suppression, irrelevant speech also eliminated the word length effect: The proportion of short words correctly recalled was .460 ; that of long words, $.452[F(1,19)<1]$.

As is also evident in Figure 1, overall performance differed among the conditions. The proportions of words correctly recalled in the quiet, irrelevant tone, irrelevant speech, and articulatory suppression conditions were $.527, .454, .456$, and .347 , respectively. Of particular interest is the finding that performance in the irrelevant tone condition was reliably worse than in the quiet condition $\left[F(1,38)=5.30, M S_{\mathrm{e}}=0.16, p<.05\right]$, and performance in the articulatory suppression condition was reliably worse than in the irrelevant speech condition $\left[F(1,38)=10.49, M S_{\mathrm{e}}=0.18, p<.01\right]$. The irrelevant tone and irrelevant speech conditions did not differ in over- 

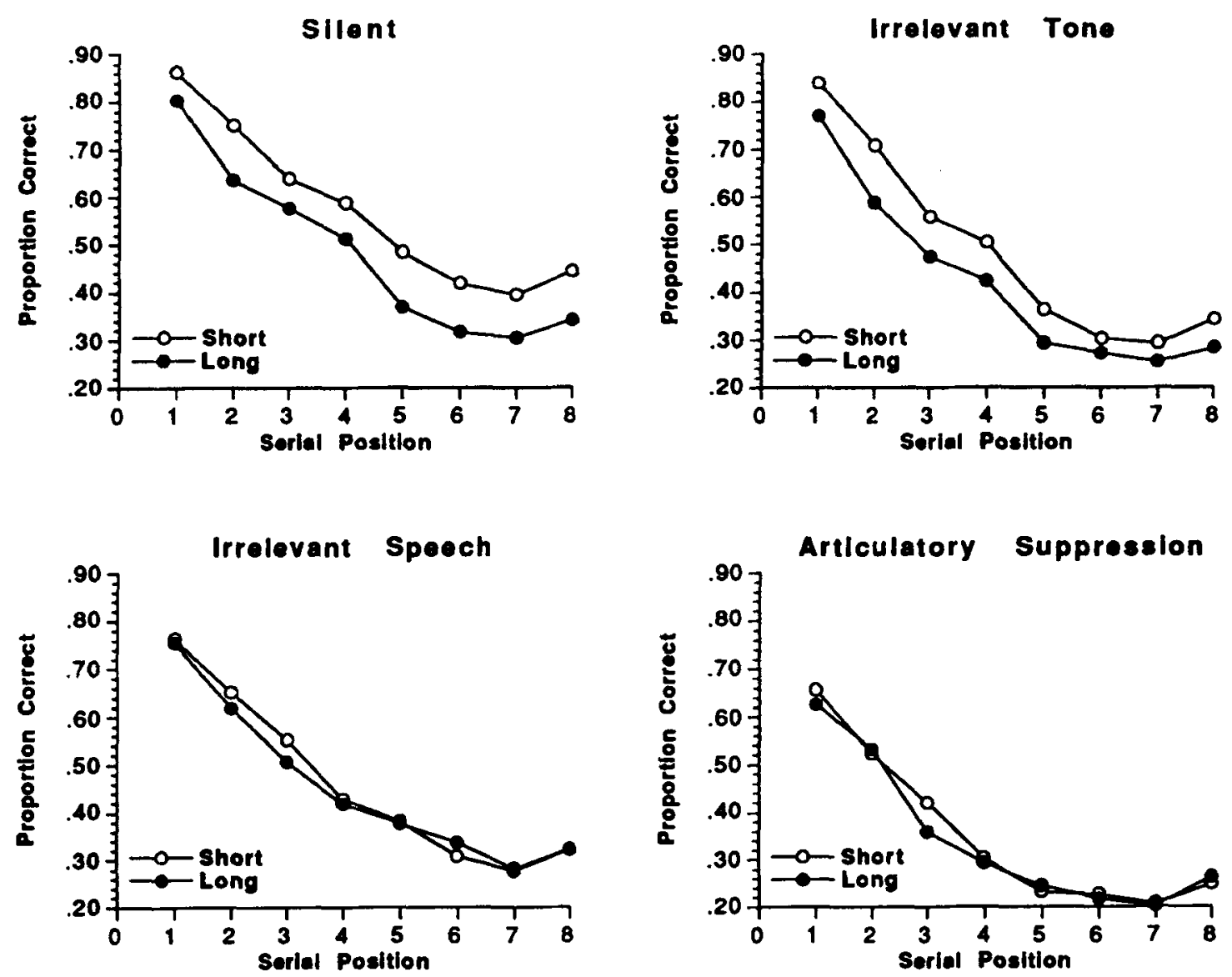

Figure 2. Proportion of short and long words correctly placed in order as a function of serial position for the four conditions in Experiment 1.

all performance, although one manipulation preserved, whereas the other eliminated, the word length effect.

Figure 2 shows the proportions of short and long words correctly recalled as a function of both background condition and serial position. In the quiet and irrelevant tone conditions, there is evidence of a word length effect of approximately equal magnitude at each position. This conclusion is supported statistically: The interaction between word length and serial position was not reliable for either condition [ for the quiet condition, $F(7,133)=$ $1.04, M S_{\mathrm{e}}=0.005, p>.40$, and for the irrelevant tone condition, $\left.F(7,133)=1.21, M S_{\mathrm{e}}=0.007, p>.30\right]$. In both the irrelevant speech and the articulatory suppression conditions, there was no evidence of an effect of word length at any position.

There was an interaction between type of irrelevant information and serial position $\left[F(21,532)=1.66, M S_{\mathrm{e}}=\right.$ $0.13, p<.05]$. This small effect is due to the differential effect of irrelevant noise versus no noise, and also of differences among the three irrelevant noise conditions. In general, the disruptive effect of irrelevant information (whether tones, irrelevant speech, or articulated speech) was greater at the beginning of the list than at the end of the list.

\section{Discussion}

Experiment 1 confirmed a novel prediction of the feature model and produced a new empirical finding: Irrelevant speech eliminates the word length effect. It has already been shown that the feature model predicts the elimination of the word length effect when subjects engage in articulatory suppression (Neath \& Nairne, 1995). The feature model offers the same explanation for both: The model conceives of both articulatory suppression and irrelevant speech as adding irrelevant information to the memory trace. This has the effect of decreasing the probability that the degraded items in primary memory will be correctly matched with the appropriate undegraded secondary memory items. Neither articulatory suppression nor irrelevant speech interferes with rehearsal; rather, they both add noise to the memory representation.

The results also have important implications for other aspects of the irrelevant speech effect. First, the results replicate those of LeCompte (1995), who found that an irrelevant speech effect was observable even when the irrelevant speech item was a repeated token. Multiple irrelevant items are not necessary for an irrelevant speech effect to be observed. Second, the results replicate those of both Jones and Macken (1993) and LeCompte, Neely, 
and Wilson (1997), who found an irrelevant tone effect: Performance in the irrelevant tone condition was impaired relative to that in the quiet condition. Third, the results suggest an important distinction between the irrelevant tone effect and the irrelevant speech effect: Although both lower overall performance, only irrelevant speech eliminates the word length effect.

Baddeley's $(1986,1992,1994)$ WMM predicted that irrelevant speech would not eliminate the word length effect because it attributes the irrelevant speech effect to interference in the phonological store and attributes the word length effect to the prevention of rehearsal in the articulatory control process. Irrelevant speech will not interfere with the articulatory control process, and so a word length effect remains. The WMM might be able to offer a post hoc explanation of why irrelevant speech eliminated the word length effect if it allowed irrelevant speech items to interfere with the articulatory control process. However, this is equivalent to saying that irrelevant speech prevents rehearsal, which seems implausible. The feature model does not require that irrelevant speech prevents rehearsal, because rehearsal is not necessary for a word length effect to be seen (see Brown \& Hulme, 1995).

This experiment also adds to other recent data that contradict the WMM explanation of the irrelevant speech effect. Both LeCompte and Shaibe (1997) and Jones and Macken (1995) have demonstrated that varying the phonological similarity between the irrelevant speech and the to-be-remembered items does not affect the magnitude of the irrelevant speech effect, thereby failing to replicate an early experiment by Salamé and Baddeley (1982). This finding is inconsistent with the WMM because working memory attributes disruption in memory to phonological confusions between the relevant and irrelevant stimuli. Furthermore, the WMM cannot explain the irrelevant tone effect, because tones carry no phonological information and so should cause no disruption in working memory.

The feature model predicted that both articulatory suppression and irrelevant speech would eliminate the word length effect. It also correctly predicted that there would be an equivalent effect of word length at each serial position. In a typical serial recall test, output interference increases over serial positions and differentially affects the long items. If a secondary memory trace is mistakenly sampled as the match for a degraded primary memory trace early in the recall process, that particular secondary memory trace is less likely to be successfully recovered. Because there are fewer intact features, on the average, for long items relative to short items, they are more likely to be mismatched early in the recall process and thus less likely to be successfully recovered when they are the correct match. As output interference is minimized, however, there is less chance of this happening, and relative to a written serial recall test, a reconstruction of order task reduces output interference.

Despite these successes, several aspects of the data were not predicted. First, although the feature model pre- dicted the elimination of the word length effect under irrelevant speech, it did not predict that there would be a difference in the overall level of recall between irrelevant speech and articulatory suppression. Because these effects are simulated in the same way, the model necessarily predicts that the results should also be the same. This difference between the conditions could be due simply to extra demands on resources when production is required as opposed to merely passive listening, but this speculation would require empirical verification. Second, the model did not predict the irrelevant tone effect, the overall reduced performance when irrelevant tones, as opposed to quiet, accompanied presentation. Although it did not predict this result, the feature model can account for it. This result could be simulated by altering the attentional parameter.

Finally, the model did not predict the interaction between the four conditions and serial position. It should be noted, however, that interactions between serial position and irrelevant speech have been inconsistent across a number of experiments. For instance, both LeCompte (1995) and LeCompte et al. (1997) found larger irrelevant speech effects at later serial positions, but LeCompte and Shaibe (1997) found larger effects at early positions in some experiments and no discernible interaction in other quite similar experiments. Salamé and Baddeley's (1982) early experiments on the irrelevant speech effect also showed surprisingly inconsistent interactions between irrelevant speech and serial position. Thus, the present interaction adds to the puzzling set of data concerning these two variables, which, to our knowledge, cannot be explained by any current theory of irrelevant speech.

\section{EXPERIMENT 2}

Experiment 1 demonstrated that irrelevant speech eliminates the word length effect, just like articulatory suppression. In that experiment, the irrelevant speech consisted of only one word, so that subjects in the irrelevant speech condition would be exposed to the same type of irrelevant information as were those in the articulatory suppression condition. Most studies on irrelevant speech, however, use multiple tokens. The purpose of Experiment 2 was to see whether irrelevant speech eliminated the word length effect when multiple irrelevant speech tokens were used. According to the feature model, there should be no functional difference between these conditions: Both a single repeated token and multiple tokens presented as irrelevant speech should contribute noise to the primary memory traces and so should eliminate the word length effect.

\section{Method}

Subjects. Thirty-six Louisiana State University undergraduates volunteered to participate in exchange for credit in introductory psychology courses. All were native speakers of American English, and each was arbitrarily assigned to one of two conditions.

Stimuli. The to-be-remembered stimuli comprised 80 of the 81 long and 80 of the 81 short words from LaPointe and Engle (1990). 
Each word occurred three times throughout the experiment. During list presentation, each word was displayed in the center of an Apple Macintosh Classic II computer screen for $1 \mathrm{sec}$ in black against a white background in 36-point Geneva font.

The to-be-ignored speech comprised 36 nonsense syllables (e.g., $v u x$ ), which were originally used by LeCompte (1994; Experiment 4). Each syllable was recorded digitally at an onset-toonset rate of $700 \mathrm{msec}$. The duration of the syllables ranged from 400 to $570 \mathrm{msec}$. The 36 syllables were arranged in a random order, and the entire sequence was repeated continually throughout the presentation and recall of each list.

Design. Sixty presentation lists were generated separately for each subject. The lists were divided into three blocks of 20 lists. Within each block, 10 lists comprising 8 items each were created by sampling randomly and without replacement from the 80 long words; the other 10 lists, also comprising 8 items each, were created by sampling randomly and without replacement from the 80 short words. Short and long lists were ordered randomly within each block. Within each block of 20 lists, a randomly determined half of the long lists and half of the short lists were presented with a quiet background - that is, in silence. The other half of the lists were presented with a speech background.

Procedure. The subjects were tested individually or in groups of up to 4 individuals. Each sat in front of a computer and wore headphones through which the irrelevant speech was played. The subjects were informed that they would see lists of words and following each list would be tested on how accurately they could remember the order in which these words had been presented.

Each list was presented at a 750 -msec rate with no blank time between words. For the lists in the background speech condition, the stream of irrelevant auditory stimuli began at approximately the same instant as the onset of the first to-be-remembered visual word and continued uninterrupted until the subject had completed recall. The relevant and irrelevant stimuli had slightly different presentation rates and were consequently asynchronous. For the lists in the quiet background condition, no sound was played through the headphones at any point during a trial.

At the end of each list, the eight list items appeared simultaneously in a new random order. They were arranged vertically in the center of the screen in 12-point Chicago font. The subjects' task was to use the mouse to click the words in the same order as that in which they had appeared on the computer screen. Unlike in Experiment 1 , as each word was clicked, it disappeared from the screen; the locations of the yet-to-be-clicked words were unaffected by this disappearance. The next list began $2 \mathrm{sec}$ after the last item was clicked. No time limit was placed on subjects during test. The experiment proper was preceded by a single practice list comprising eight words of intermediate length presented with no background speech.

\section{Results and Discussion}

Experiment 2 nicely replicated the results of Experiment 1 , as the top panel of Figure 3 shows. With a quiet background, the proportion of words ordered correctly was .426 compared to .369 with a speech background $\left[F(1,35)=26.04, M S_{\mathrm{e}}=0.037, p<.01\right]$. In the quiet condition, a reliable word length effect obtained: The proportion of short words (.448) ordered correctly was greater than the proportion of long words $(.404)$ ordered correctly $\left[F(1,35)=6.24, M S_{\mathrm{e}}=0.045, p<.05\right]$. However, in the irrelevant speech condition, there was no measurable word length effect: The proportion of short words correctly ordered was .365 compared to .372 for long words $[F(1,35)<1]$. Just like articulatory suppres-
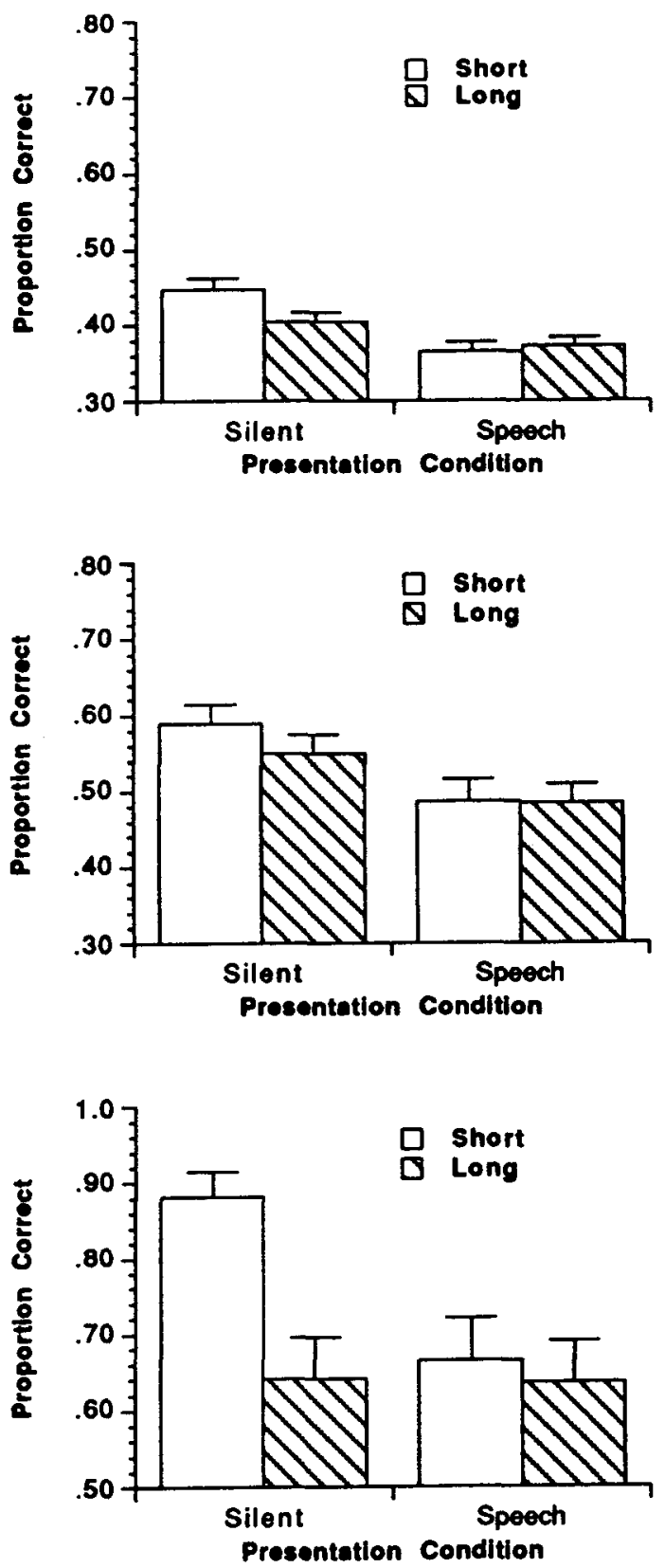

Figure 3. Proportion of short and long words correctly recalled in a serial reconstruction task as a function of background condition (quiet or irrelevant speech) when the irrelevant speech comprised 36 different items (Experiment 2, top panel), when presentation was auditory rather than visual (Experiment 3, middle panel), and when written serial recall was required rather than reconstruction of order (Experiment 4, bottom panel). Error bars show the standard error of the mean.

sion, irrelevant speech eliminates the word length effect for visually presented items, even when the irrelevant speech is composed of multiple different items.

There was no overall advantage in performance of short over long words $[.407$ vs. .388 , respectively; $F(1,35)$ 

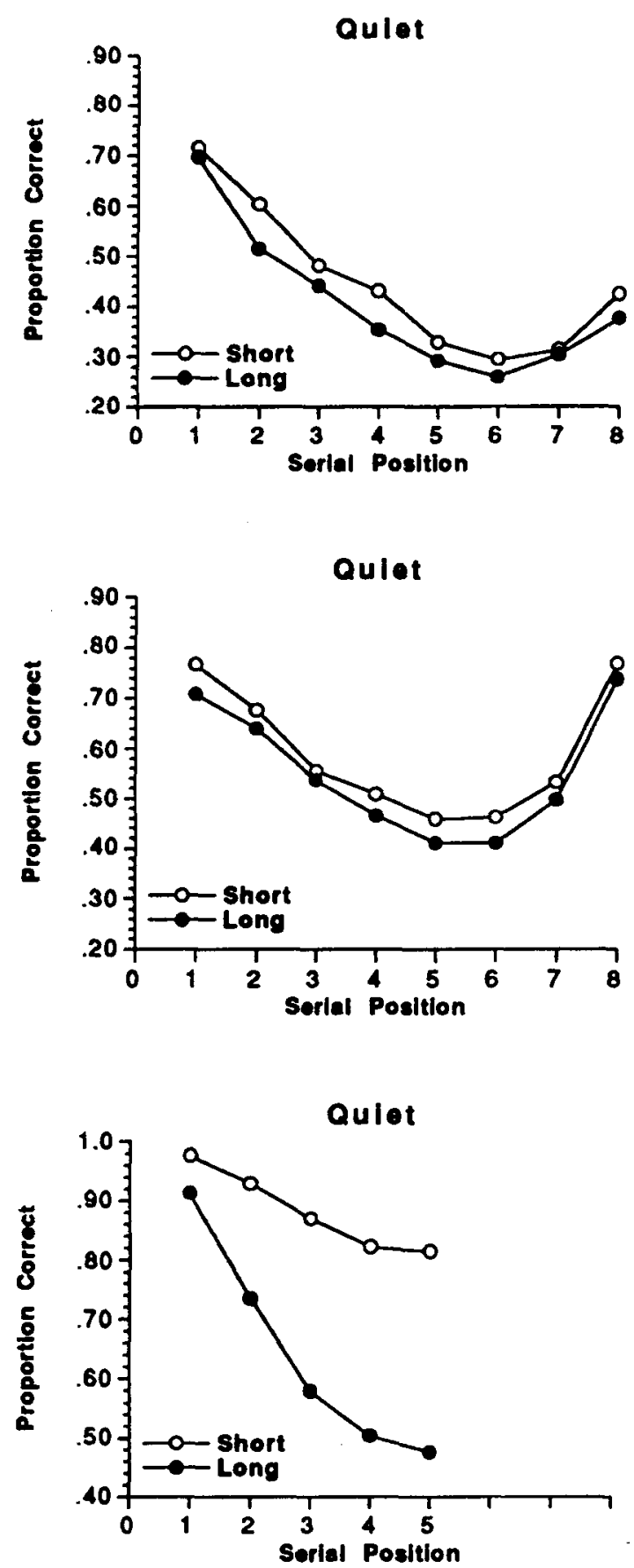
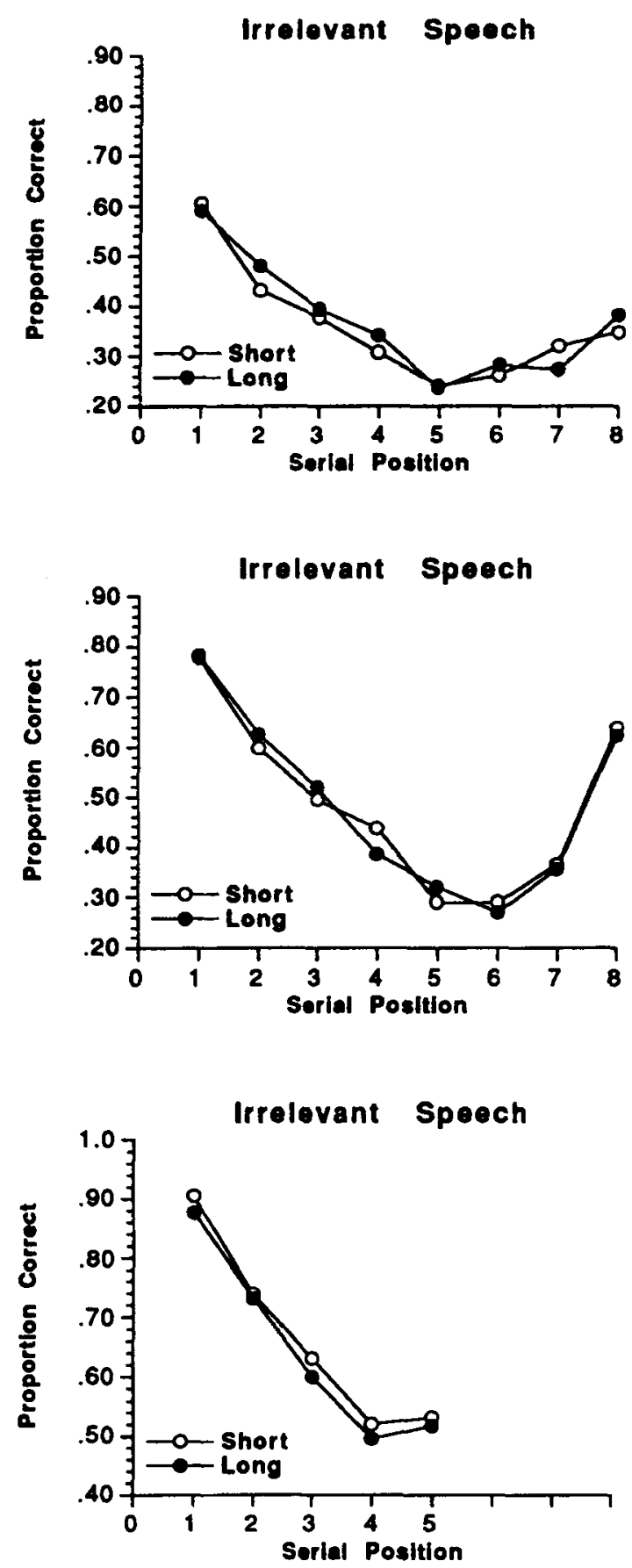

Figure 4. Proportion of short and long words correctly placed in order as a function of serial position and background condition for Experiment 2 (top panels), Experiment 3 (middle panels), and Experiment 4 (bottom panels).

$\left.=1.84, M S_{\mathrm{e}}=0.053, p>.15\right]$, but there was a reliable interaction between word length and background condition $\left[F(1,35)=4.23, M S_{\mathrm{e}}=0.045, p<.05\right]$, as is described above and shown in the top panel of Figure 3. There was a typical main effect of serial position $[F(7,245)$ $\left.=61.73, M S_{\mathrm{e}}=0.040, p<.01\right]$, but there was no reliable interaction between word length and serial position $[F(7,245)<1]$. This lack of interaction between word length and serial position, shown in the top panel of Fig- ure 4, replicates the results of Experiment 1 . The size of the word length effect was equivalent at each position in the quiet condition and was absent at each serial position in the irrelevant speech condition.

There was a reliable interaction between background condition and serial position $\left[F(7,245)=3.32, M S_{e}=\right.$ $0.015, p<.01]$. The disruptive effect of irrelevant speech was greater at the beginning of the list than at the end of the list. Finally, there was a reliable three-way interac- 
tion between word length, background condition, and serial position $\left[F(7,245)=2.16, M S_{\mathrm{e}}=0.011, p<.05\right]$, due primarily to a larger two-way interaction for the short words. As in Experiment 1, the feature model did not predict that irrelevant speech would interact with serial position, but neither did any other theory. The important result, however, is the replication of the main result of Experiment 1: Irrelevant speech eliminated the word length effect.

\section{EXPERIMENT 3}

In Experiments 1 and 2, irrelevant speech eliminated the word length effect for visually presented items, supporting the idea that its effects are like those of articulatory suppression. It did so, moreover, regardless of whether the irrelevant speech consisted of a single repeating item (Experiment 1) or a large pool of items (Experiment 2). Given that the feature model represents the effects of articulatory suppression and irrelevant speech in the same way, it predicts that irrelevant speech will also eliminate the word length effect for auditory items because articulatory suppression eliminates the word length effect for auditory items (Baddeley et al., 1984). In Experiment 3, we tested this prediction.

\section{Method}

Subjects. Thirty-six Purdue University undergraduates volunteered to participate in exchange for credit in introductory psychology courses. All were native speakers of American English, and each was arbitrarily assigned to one of two conditions.

Stimuli. The to-be-remembered stimuli were the 81 long and 81 short words used by LaPointe and Engle (1990). Each word was digitized at $22 \mathrm{kHz}$ on an Apple Macintosh IIci computer by a male speaker. The to-be-ignored speech was the word teatime digitized by the same male speaker at a rate of $22 \mathrm{kHz}$. This was the same token used in Experiment 1. The irrelevant speech began immediately prior to the first trial and continued uninterrupted throughout both presentation and recall until the end of the experiment.

Design. As in Experiment 1, irrelevant speech was manipulated between subjects. Thus, 60 presentation lists were generated separately for each subject. The lists were divided into three blocks of 20 lists. Within each block, 10 lists comprising 8 items each were created by sampling randomly and without replacement from the 81 long words; the other 10 lists, also comprising 8 items each, were created by sampling randomly and without replacement from the 81 short words. Short and long lists were ordered randomly within each block. For the subjects in the quiet condition, all 60 lists were presented without irrelevant speech; for the subjects in the irrelevant speech condition, all 60 lists were presented with irrelevant speech.

Procedure. The subjects were tested individually or in pairs. Each sat in front of an Apple PowerPC 6100 computer and wore headphones through which all stimuli were played. The subjects were informed that they would hear lists of words and following each list would be tested on how accurately they could remember the order in which these words had been presented. Each token was presented with a stimulus onset asynchrony of $1,500 \mathrm{msec}$, and there was no visual component. For the lists in the background speech condition, the stream of irrelevant auditory stimuli began prior to presentation of the first list and continued uninterrupted until the subject had completed the experiment. The relevant and irrelevant stimuli had different presentation rates and were consequently asynchronous.

At the end of each list, the eight list items appeared simultaneously in alphabetical order. They were arranged horizontally in the center of the screen in 12-point Chicago font. The subject's task was to use the mouse to click on the words in the same order as that in which they had appeared on the computer screen. Unlike in Experiment 2 , the word did not disappear as it was clicked. The next list began $2 \mathrm{sec}$ after the last item from the previous list was clicked. No time limit was placed on subjects during test. The experiment proper was preceded by a single practice list comprising eight digits (1-8) presented with no background speech in the quiet condition and with background speech in the irrelevant speech condition.

\section{Results and Discussion}

As the middle panel of Figure 3 shows, Experiment 3 replicated the findings of Experiments 1 and 2 that irrelevant speech eliminates the word length effect, but this time for auditory items. In the quiet condition, a reliable word length effect obtained, replicating the finding of a word length effect in a reconstruction of order task seen in Experiments 1 and 2 and extending it to lists of auditory items. The proportion of short words $(.590)$ ordered correctly was greater than the proportion of long words $(.548)$ ordered correctly $\left[F(1,17)=9.28, M S_{\mathrm{e}}=\right.$ $0.014, p<.01]$. However, in the irrelevant speech condition, there was no measurable word length effect: The proportion of short words (.486) correctly ordered was the same as the proportion of long words (.485) correctly ordered $[F(1,17)<1]$. This finding is consistent with the prediction of the feature model but counter to the predictions of the WMM: Just as articulatory suppression eliminates the word length effect for both visual and auditory presentation, irrelevant speech also eliminates the word length effect for both presentation modalities. ${ }^{3}$

With a quiet background, the proportion of words ordered correctly was .569 compared to .486 when there was a speech background $\left[F(1,34)=5.82, M S_{\mathrm{e}}=0.169\right.$, $p<.05]$. Irrelevant speech thus impairs memory for auditory items just as it impairs memory for visual items. This finding replicates those from the few studies in which researchers have explored the effects of irrelevant speech on auditory stimuli (Hanley \& Broadbent, 1987; LeCompte, 1996; Salamé \& Baddeley, 1982).

Unlike in Experiments 1 and 2, there was an overall advantage in performance of short over long words $(.538$ vs. .517 , respectively; $F(1,34)=4.30, M S_{\mathrm{e}}=0.016, p<$ $.05]$. This was qualified by a reliable interaction between word length and background condition $[F(1,34)=4.21$, $\left.M S_{\mathrm{e}}=0.016, p<.05\right]$, as is described above and shown in the middle panel of Figure 3. There was a typical main effect of serial position $\left[F(7,238)=92.47, M S_{\mathrm{e}}=0.018\right.$, $p<.01]$, but just as in Experiment 1, there was no reliable interaction between word length and serial position $[F(7,238)<1]$. This lack of interaction, shown in the middle panel of Figure 4, replicates what was found in the previous two experiments. Comparison of the top and middle panels of Figure 4 shows that Experiment 3 
also resulted in a modality effect, enhanced recall of the final items when the presentation modality was auditory rather than visual. This study thus replicates the finding that the modality effect is observed in a reconstruction of order task (Neath, 1997).

Finally, there was a reliable interaction between background condition and serial position $[F(7,238)=4.45$, $\left.M S_{\mathrm{e}}=0.018, p<.05\right]$. The disruptive effect of irrelevant speech was larger for the middle serial positions than for the early serial positions. Note that this pattern is different from that found in the two previous experiments, and it emphasizes the variability of the interaction. It may be attributable to the change in presentation modality which led to a modality effect. Unlike in Experiment 2 , however, the three-way interaction was not reliable $[F(7,238)<1]$.

\section{EXPERIMENT 4}

In Experiment 3, irrelevant speech eliminated the word length effect for auditory items, suggesting that the results of Experiment 1 and 2 may be quite general. However, in all three experiments so far, we used a large pool of tobe-remembered items, a relatively long list length, and a reconstruction of order task. It is possible that the results described above are unique to this situation and would not be observable with other testing procedures. The purpose of Experiment 4 was to replicate the finding that irrelevant speech eliminates the word length effect in a setting more similar to those used in developing the WMM in the first place (e.g., Baddeley et al., 1984; Baddeley et al., 1975). Thus, as in Baddeley's work, there was a small pool of to-be-remembered words, the lists were only five items long, and written serial recall was required.

\section{Method}

Subjects. Eighteen Purdue University undergraduates volunteered to participate in exchange for credit in introductory psychology courses. All were native speakers of American English.

Stimuli. The to-be-remembered stimuli comprised eight long and eight short words, from the small pool previously used by LaPointe and Engle (1990); these words were part of the larger pools used in the three previous experiments. The long words were alcohol, amplifier, gallery, mosquito, musician, officer, orchestra, and property; the short words were beast, bronze, dirt, golf, inn, lump, star, wife. Baddeley's stimuli (e.g., Baddeley et al., 1984, Baddeley et al., 1975) were not used because many of those items were not familiar to the local subject population. During list presentation, each word was displayed in the center of a computer monitor for $1 \mathrm{sec}$ in black against a white background in 18-point bold Geneva font. The to-be-ignored speech stimulus was the same as in Experiments 1 and 3.

Design. Fifty-six presentation lists were generated randomly for each subject. The lists were divided into two blocks of 28 lists. Within each block, 14 lists comprising five items each were created by sampling randomly from the 8 long words; the other 14 lists, also comprising five items each, were created by sampling randomly from the eight short words. The only other constraint was that no word could occur more than once in any list. Short and long lists were ordered randomly within each block.
For half of the subjects, the first block of 28 lists were presented with a quiet background and the second block was presented with irrelevant speech. For the remaining subjects, this order was reversed. A preliminary analysis of variance showed no main effect of presentation order and no interaction with other variables of interest; the data were collapsed over this variable for all subsequent analyses.

Procedure. The subjects were tested individually or in pairs. Each sat in front of an Apple PowerPC 6100 computer and wore headphones through which the warning tone and irrelevant speech were played. The subjects were informed that they would see lists of words and following each list would be tested on how accurately they could remember the order in which these words had been presented.

Each word was displayed for $1 \mathrm{sec}$ with a 500 -msec interstimulus interval. For the lists in the background speech condition, the stream of irrelevant auditory stimuli began at the beginning of the block and continued uninterrupted until the end of the block. For the lists in the quiet background condition, no sound was played through the headphones at any point during a trial, with the exception of a warning beep that occurred $1 \mathrm{sec}$ prior to the beginning of each list.

At the end of each list, the screen went blank and the subject wrote down the words in strict serial order on an answer sheet. An experimenter ensured that strict serial recall instructions were followed; one additional subject's data were eliminated for the subject's having failed to follow recall instructions. No time limit was placed on subjects during test, and the subjects were told that misspellings were not important. The experiment proper was preceded by a single practice list comprising five words of varying length presented with no background speech.

\section{Results and Discussion}

As the bottom panel of Figure 3 shows, Experiment 4 replicated the finding that irrelevant speech eliminates the word length effect even when the list length is shortened, the pool of to-be-remembered words is smaller, and written serial recall is required. In the quiet condition, a reliable word length effect obtained: The proportion of short words $(.882)$ recalled correctly was greater than the proportion of long words $(.641)$ recalled correctly $\left[F(1,17)=89.98, M S_{\mathrm{e}}=0.006, p<.01\right]$. However, in the irrelevant speech conditions, there was no measurable effect of word length: The proportion of short words correctly ordered was .665 ; that of the long words, $.644\left[F(1,17)=2.57, M S_{\mathrm{e}}=0.002, p>.10\right]$.

With a quiet background, the proportion of words recalled was .762; it was .655 with a speech background $\left[F(1,17)=23.61, M S_{\mathrm{e}}=0.044, p<.01\right]$. This demonstrates the main effect of irrelevant speech. There was an overall advantage in recall of short over long words [.774 vs. . 640 , respectively, $F(1,17)=72.78, M S_{\mathrm{e}}=0.021, p<$ $.01]$, and there was a reliable interaction between word length and background condition $\left[F(1,17)=69.39, M S_{\mathrm{e}}=\right.$ $0.016, p<.01]$, as is described above and shown in the bottom panel of Figure 3 . There was a typical main effect of serial position $\left[F(4,68)=58.02, M S_{\mathrm{e}}=0.025, p<.01\right]$.

Unlike in the previous three experiments, there was a reliable interaction between word length and serial position $\left[F(4,68)=4.26, M S_{\mathrm{e}}=0.013, p<.01\right]$. This is shown in the bottom panel of Figure 4 . This interaction 
is predicted by the feature model, because output interference increases with written serial recall and affects long items more than it affects short items (see Table 6 of Neath \& Nairne, 1995). Also unlike in the previous experiments, there was no interaction between background condition and serial position $\left[F(4,68)=1.56, M S_{\mathrm{e}}=\right.$ $0.016, p>.19]$. The three-way interaction was reliable $\left[F(4,68)=4.54, M S_{\mathrm{e}}=0.013, p<.01\right]$.

There were several differences between the results of Experiment 4 (with strict serial recall of a small pool of items from a short list) and Experiments 1, 2, and 3 (with reconstruction of order of a large pool of items from a longer list). First, there was an interaction between word length and serial position in Experiment 4, which replicates previous findings with this paradigm (e.g., Cowan et al., 1992). Second, there was a reliable interaction between background condition and serial position in Experiments 1,2, and 3, but no such interaction in Experiment 4 . Another difference concerns the magnitude of the basic word length effect in the quiet conditions. This difference was far larger in Experiment 4 than in any of the previous experiments, although the difference was always reliable. This increase in the size of the effect may be attributable to the extra role of output time (see, e.g., Brown \& Hulme, 1995; Cowan et al., 1992).

Even with these minor differences, Experiment 4 replicated and extended the important finding from Experiments 1, 2, and 3: Even when a small pool of to-beremembered items is presented in a short list with immediate written serial recall, irrelevant speech eliminates the word length effect. Thus, the prediction of the feature model applies to more than just one paradigm, list length, pool size, and test method.

\section{GENERAL DISCUSSION}

The current version of the WMM was designed to explain four basic findings: the phonological similarity effect, the word length effect, the irrelevant speech effect, and the effect of articulatory suppression (Baddeley, 1986, $1992,1994)$. The explanation of the phonological similarity effect is not really different from that offered by other theories, except that the WMM places the location in the phonological store. Previous experiments have already documented problems with the WMM's explanation for the irrelevant speech effect (e.g., Jones \& Macken, 1993, 1995; LeCompte \& Shaibe, 1997; Martin, 1993), articulatory suppression (Howard \& Butterworth, 1989; Margolin, Griebel, \& Wolford, 1982), and the word length effect (e.g., Brown \& Hulme, 1995; Caplan, Rochon, \& Waters, 1992; Neath \& Nairne, 1995).

The experiments reported here compound this problem: The WMM makes an incorrect prediction about the interaction between two of the core phenomena that it was designed to explain, word length and irrelevant speech. Although the WMM has been applied to many different areas as a general heuristic, it is worrisome that many predictions involving the four phenomena that it was designed to explain are incorrect. If the WMM is amended to account in a post hoc fashion for the present results, it entails the added assumption that irrelevant speech prevents rehearsal.

The feature model was designed originally to explain recency effects in immediate memory and has been extended to account for many other phenomena, including modality effects, suffix effects, grouping effects, phonological similarity effects, stimulus discriminability effects, and the effects of word length, articulatory suppression, and irrelevant speech (Nairne, 1990; Neath \& Nairne, 1995; Surprenant \& Neath, 1996). What is perhaps most encouraging about the model is its ability to make novel predictions concerning interactions between these factors. For example, it correctly predicted that suffix effects obtain under both articulatory suppression and irrelevant speech (Surprenant et al., 1997) and, in the present experiments, that irrelevant speech will eliminate the word length effect. Note that these predictions hold regardless of parameter settings: Using the same default set of parameters, the model requires that these effects obtain. Just like the WMM, the feature model is by no means perfect; Nairne (1990) and Neath and Nairne (1995) have documented several fundamental limitations, and several others have been noted in connection with the results of Experiment 1.

Both the WMM (Baddeley, 1986, 1992, 1994) and the feature model (Nairne, 1990; Neath \& Nairne, 1995) offer explanations for the effects of word length, articulatory suppression, and irrelevant speech. In particular, both describe how each of these should-or should notinteract. If the two views are compared in their ability to explain these basic memory processes and their interactions, one finds that the explanation offered by the feature model works, and the one offered by working memory does not.

\section{REFERENCES}

Baddeley, A. D. (1986). Working memory. New York: Oxford University Press.

Baddeley, A. D. (1992, January 31). Working memory. Science, 255, 556-559.

BADDELEY, A. D. (1994). Working memory: The interface between memory and cognition. In D. L. Schacter \& E. Tulving (Eds.), Memory systems 1994 (pp. 351-367). Cambridge, MA: MIT Press.

BADDELEY, A. [D.], \& Lewis, V. [J.] (1984). When does rapid presentation enhance digit span? Bulletin of the Psychonomic Society, 22, 403-405.

Baddeley, A. D., Lewis, V. J., \& VAllar, G. (1984). Exploring the articulatory loop. Quarterly Journal of Experimental Psychology, 36, 233-252.

Baddeley, A. D., Thomson, N., \& Buchanan, M. (1975). Word length and the structure of short-term memory. Journal of Verbal Learning \& Verbal Behavior, 14, 575-589.

Brown, G. D. A., \& Hulme, C. (1995). Modeling item length effects in memory span: No rehearsal needed? Journal of Memory \& Language, 34, 594-621. 
Caplan, D., Rochon, E., \& Waters, G. S. (1992). Articulatory and phonological determinants of word-length effects in span tasks. Quarterly Journal of Experimental Psychology, 45A, 177-192.

Colle, H. A., \& Welsh, A. (1976). Acoustic masking in primary memory. Journal of Verbal Learning \& Verbal Behavior, 15, 17-32.

ConRad, R. (1964). Acoustic confusions in immediate memory. British Journal of Psychology, 55, 75-84.

CowAN, N. (1995). Attention and memory: An integrated framework. New York: Oxford University Press.

Cowan, N., Cartwright, C., Winterowd, C., \& Sherk, M. (1987). An adult model of preschool children's speech memory. Memory \& Cognition, 15, 511-517.

Cowan, N., Day, L., Saults, J. S., Keller, T. A., Johnson, T., \& FloRES, L. (1992). The role of verbal output time in the effects of word length on immediate memory. Journal of Memory \& Language, 31 , 1-17.

HANLEY, J. R., \& BRoADBENT, C. (1987). The effect of unattended speech on serial recall following auditory presentation. British Journal of Psychology, 78, 287-297.

Howard, D., \& ButTerworth, B. (1989). Developmental disorders of verbal short-term memory and their relation to sentence comprehension: A reply to Vallar and Baddeley. Cognitive Neuropsychology, 6, 455-463.

JONES, D. M., \& MACKEN, W. J. (1993). Irrelevant tones produce an irrelevant speech effect: Implications for phonological coding in working memory. Journal of Experimental Psychology: Learning, Memory, \& Cognition, 19, 369-381.

Jones, D. M., \& Macken, W. J. (1995). Phonological similarity in the irrelevant speech effect: Within- or between-stream similarity? Journal of Experimental Psychology: Learning, Memory, \& Cognition, 21, 103-115.

LAPointe, L. B., \& ENGLE, R. W. (1990). Simple and complex word spans as measures of working memory capacity. Journal of Experimental Psychology: Learning, Memory, \& Cognition, 16, 1118 1133.

LECOMPTE, D. C. (1994). Extending the irrelevant speech effect beyond serial recall. Journal of Experimental Psychology: Learning, Memory, \& Cognition, 20, 1396-1408.

LECOMPTE, D. C. (1995). An irrelevant speech effect with repeated and continuous background speech. Psychonomic Bulletin \& Review, 2, 391-397.

LECOMPTE, D. C. (1996). Irrelevant speech, serial rehearsal, and temporal distinctiveness: A new approach to the irrelevant speech effect. Journal of Experimental Psychology: Learning, Memory, \& Cognition, 22, 1154-1165.

LeCompte, D. C., Neely, C. B., \& Wilson, J. R. (1997). Irrelevant speech and irrelevant tones: The relative importance of speech to the irrelevant speech effect. Journal of Experimental Psychology: Learning, Memory, \& Cognition, 23, 472-483.

LeCompte, D. C., \& Shaibe, D. M. (1997). On the irrelevance of phonology to the irrelevant speech effect. Quarterly Journal of Experimental Psychology, 50A, 100-118.

Longoni, A. M., Richardson, J. T. E., \& Aiello, A. (1993). Articulatory rehearsal and phonological storage in working memory. Memory \& Cognition, 21, 11-22.

LucE, R. D. (1963). Detection and recognition. In R. D. Luce, R. R. Bush, \& E. Galanter (Eds.), Handbook of mathematical psychology (pp. 103-189). New York: Wiley.

MACKEN, W. J., \& Jones, D. M. (1995). Functional characteristics of the inner voice and the inner ear: Single or double agency? Journal of Experimental Psychology: Learning, Memory, \& Cognition, 21, 436-448

MACKwORTH, J. F. (1963). The duration of the visual image. Canadian Journal of Psychology, 17, 62-81.
Margolin, C. M., Griebel, B., \& Wolford, G. (1982). Effect of distraction on reading versus listening. Journal of Experimental Psychology: Learning, Memory, \& Cognition, 8, 613-618.

MarTin, R. C. (1993). Short-term memory and sentence processing: Evidence from neuropsychology. Memory \& Cognition, 21, 176183.

Melton, A. W. (1963). Implications of short-term memory for a general theory of memory. Journal of Verbal Learning \& Verbal Behavior, 2, 1-21.

MURRAY, D. J. (1968). Articulation and acoustic confusability in shortterm memory. Journal of Experimental Psychology, 78, 679-684.

NAIRNE, J. S. (1988). A framework for interpreting recency effects in immediate serial recall. Memory \& Cognition, 16, 343-352.

NAIRNE, J. S. (1990). A feature model of immediate memory. Memory \& Cognition, 18, 251-269.

NeaTh, I. (1997). Modality, concreteness, and set-size effects in a free reconstruction of order task. Memory \& Cognition, 25, 256-263.

NeATH, I., \& NAIRNE, J. S. (1995). Word-length effects in immediate memory: Overwriting trace decay theory. Psychonomic Bulletin \& Review, 2, 429-441.

SALAMÉ, P., \& BADDeley, A. D. (1982). Disruption of short-term memory by unattended speech: Implications for the structure of working memory. Joumal of Verbal Learning \& Verbal Behavior, 21, 150-164.

Surprenant, A. M., LeCompte, D. C., \& Neath, I. (1997). Manipulations of irrelevant information: Suffix effects with articulatory suppression and irrelevant speech. Manuscript in preparation.

Surprenant, A. M., \& Neath, I. (1996). The relation between discriminability and memory for vowels, consonants, and silent-center vowels. Memory \& Cognition, 24, 356-366.

Watkins, M. J. (1972). Locus of the modality effect in free recall. Journal of Verbal Learning \& Verbal Behavior, 11, 644-648.

WATKINS, M. J., \& WATKINS, O. C. (1973). The postcategorical status of the modality effect in serial recall. Journal of Experimental Psychology, 99, 226-230.

\section{NOTES}

1. We focus on Baddeley's (e.g., Baddeley 1986, 1992, 1994) version of working memory (indicated by working memory model, or WMM) rather than the more generic idea of a working memory because only the former is specified in sufficient detail to make a prediction as compared with a post hoc explanation (see, e.g., Cowan, 1995).

2 . It is important to note what prediction means in the context of the feature model. Prediction means that regardless of the particular parameter settings, the feature model produces the same output. Thus, regardless of the values of individual parameters, simulation results from the feature model always show that irrelevant speech will eliminate the word length effect.

3. One might be tempted to compare Experiments 2 and 3 (or Experiment 1 and 3 ) in order to see whether the magnitude of the word length effect is larger for visual than for auditory items, as the feature model predicts (Neath \& Nairne, 1995). However, this is not an appropriate comparison; because the experiments were not designed to test this prediction, there are numerous differences between the experiments. Nonetheless, the numerical differences are in the correct direction. For Experiment 1 (visual): .767 (short) -.707 (long) $=.060$. For Experiment 2 (also visual): $.448-.404=.044$. Both of these are numerically larger than the value for Experiment 3 (auditory): .590$.548=.042$.

(Manuscript received November 19, 1996; revision accepted for publication February 10, 1997.) 